Appl. Phys 2, 123-128 (1973)

(C) by Springer-Verlag 1973

\title{
Electromagnetic Field Representation in Inhomogeneous Anisotropic Media *
}

\author{
A. Mohsen \\ Radiation Laboratory, The University of Michigan, Ann Arbor, Michigan 48105, USA
}

Received 9 April 1973 / Completed 21 June 1973

\begin{abstract}
Some of the basic developments in the theory of electromagnetic field representation in terms of Hertz vectors are reviewed. A solution for the field in an inhomogeneous anisotropic medium is given in terms of the two Hertz vectors. Conditions for presentation of the field in terms of uncoupled transverse electric and transverse magnetic modes, in a general orthogonal coordinate system, are derived when the permeability and permittivity tensors have only diagonal components. These conditions are compared with some known special cases.
\end{abstract}

Index Headings: Electromagnetic theory - Anisotropic medium - Hertzian-vector representation

Analysis of electromagnetic field in inhomogeneous anisotropic media has practical applications in antenna, scattering and propagation problems. In the analysis of radiation from an oscillating electric dipole in free space, Hertz [1] introduced a potential of the type which now bears his name. Since that time, considerable use has been made of the Hertz potentials. Field representation, using electric and magnetic Hertzian potentials was investigated by Whittaker [2], Debye [3], Bromwich [4] and Hansen [5]. Extension of Hansen's work for radially inhomogeneous media of constant permeability is due to Bremmer [6] and Tai [7]. A general theory of the Hertz vectors and the associated gauge transformation for inhomogeneous anisotropic media was developed by Nisbet [8], [9] and this was presented in 4-vector form by McCrea [10].

The Whittaker and Debye-Bromwich types of representation, which split the field into so-called transverse electric (TE) and transverse magnetic (TM) parts, have had considerable and fruitful applications for homogeneous media. The search

* This work was supported by the National Aeronautics and Space Administration, Langley Research Center, under NASA Grant NGR-23-005-477. for situations giving rise to uncoupled TE and TM fields is justified by the fact that, in such cases, the calculations involved can be considerably simplified. Such situations were investigated in terms of general orthogonal coordinates in homogeneous media by Bromwich [4], by Nisbet [9] for isotropic inhomogeneous media in the presence of sources, and by Friedman [11] for inhomogeneous anisotropic media when the permeability is constant. Some special cases were discussed by Wexler [12] in connection with mode uncoupling in inhomogeneously loaded rectangular and circular waveguides and by Uslenghi [13] for the radially inhomogeneous cylinder. The error in the latter work was pointed out by Parkinson [14]. Mode uncoupling via transformation of coordinates for the radially inhomogeneous cylindrical plasma column was recently considered by Bisbing [15]. Benenson and Zaytsev [16] considered mainly homogeneous anisotropic media and discussed the propagation of TEM, TE and TM waves in cylindrical and spherical coordinate systems. Mention should also be made of contributions by Luneberg [17], Marcuvitz [18], Green and Wolf [19], Samaddar [20], and Felsen and Marcuvitz [21]. 
In spite of these developments, a full treatment of the question of mode uncoupling is not yet available even for homogeneous media. Although the present paper does not resolve this general question, it does treat a particular anisotropic model. The media under consideration are assumed source free. It is known that in many applications, especially when mixed boundary conditions are to be applied, mode coupling is necessary in order to satisfy such conditions. These cases are not considered in the present work and the possibility of mode uncoupling by transformation of coordinates will not be discussed. A general solution of Maxwell equations for sourcefree anisotropic inhomogeneous media is presented in terms of general orthogonal coordinates. The permittivity $\tilde{\varepsilon}$ and permeability $\tilde{\boldsymbol{\mu}}$ are tensor functions of position, assumed non-singular so that their reciprocal exist. The gauge transformations allow for field representation in terms of only two scalars (components of Hertzian potentials) satisfying certain differential equations. These equations are, in general, of higher order than the second, but under certain conditions they reduce to second order. These conditions, which lead to uncoupled TE and TM representations, are considered in detail when the permittivity and permeability have their principal axes in the direction of the coordinate axes. The previous works of Bromwich [4], Nisbet [9], Wexler [12], Parkinson [14] and Friedman [11] are special cases of the present analysis.

\section{Field Equations}

The electromagnetic field within a stationary nonconducting source-free medium satisfies Maxwell's equations

$$
\begin{aligned}
& \nabla \times \boldsymbol{E}=-\dot{\boldsymbol{B}} \\
& \nabla \times \boldsymbol{H}=\dot{\boldsymbol{D}} \\
& \nabla \cdot \boldsymbol{B}=0 \\
& \nabla \cdot \boldsymbol{D}=0
\end{aligned}
$$

and the constitutive relations

$$
\begin{aligned}
& D=\tilde{\varepsilon} \cdot \boldsymbol{E} \\
& \boldsymbol{B}=\tilde{\boldsymbol{\mu}} \cdot \boldsymbol{H},
\end{aligned}
$$

where a dot over a function indicates derivative with respect to time, and the permittivity $\tilde{\varepsilon}$ and permeability $\tilde{\boldsymbol{\mu}}$ are tensor functions of position and are assumed non-singular so that their reciprocals exist.

Since $\boldsymbol{B}$ is a selonoidal vector, one may write

$\boldsymbol{B}=\boldsymbol{V} \times \dot{\pi}_{e}$

where $\pi_{e}$ is a Hertz vector (electric type). Using (7), Eqs. (1) to (6) lead to

$D=\nabla \times\left(\tilde{\mu}^{-1} \cdot \nabla \times \pi_{e}\right)$

and

$\tilde{\boldsymbol{\varepsilon}} \cdot \ddot{\boldsymbol{\pi}}_{e}+\nabla \times\left(\tilde{\boldsymbol{\mu}}^{-1} \cdot \nabla \times \boldsymbol{\pi}_{e}\right)=\tilde{\boldsymbol{\varepsilon}} \cdot \nabla \Psi_{e}$

where $\Psi_{e}$ is an auxiliary function which provides some flexibility in the choice of $\pi_{e}$, according to gauge transformation.

A similar analysis, due to the duality between electric and magnetic quantities, yields

$$
\begin{aligned}
& \boldsymbol{D}=-\nabla \times \dot{\boldsymbol{\pi}}_{m} \\
& \boldsymbol{B}=\nabla \times\left(\tilde{\boldsymbol{\varepsilon}}^{-1} \cdot \nabla \times \boldsymbol{\pi}_{m}\right)
\end{aligned}
$$

and

$\tilde{\boldsymbol{\mu}} \cdot \dot{\pi}_{m}+\nabla \times\left(\tilde{\boldsymbol{\varepsilon}}^{-1} \cdot \nabla \times \pi_{m}\right)=\tilde{\boldsymbol{\mu}} \cdot \nabla \Psi_{m}$.

A more general representation of the field may be obtained in terms of both electric $\left(\boldsymbol{\pi}_{e}\right)$ and magnetic $\left(\pi_{m}\right)$ Hertz vectors in the form

$\boldsymbol{B}=\nabla \times \dot{\pi}_{e}+\nabla \times\left(\tilde{\boldsymbol{\varepsilon}}^{-1} \cdot \nabla \times \pi_{m}\right)$

and

$D=-\nabla \times \dot{\pi}_{m}+\nabla \times\left(\tilde{\boldsymbol{\mu}}^{-1} \cdot \nabla \times \pi_{e}\right)$.

The question of generality of the above representation was considered by Nisbet [8], [9] who derived identical equations starting from a representation in terms of scalar and vector potentials.

It may be pointed out that the introduction of both types of Hertzian vectors is for the sake of generality, and may facilitate the analysis because of duality between electric and magnetic parameters, and depending upon the particular problem under consideration, one type may be more appropriate than the other. The fact that one type leads to the other may be made obvious by introducing a new Hertz vector $\boldsymbol{\Pi}_{e}$ given by

$\dot{\Pi}_{e}=\dot{\pi}_{e}+\tilde{\varepsilon}^{-1} \cdot \nabla \times \pi_{m}$.

Upon using (8) and (12), Eq. (14) immediately follows. In fact, since the solution of Maxwell's equation is uniquely determined by assigning initial 
values to the six components of $\boldsymbol{E}$ and $\boldsymbol{H}$ at $t=0$, which in the meantime must satisfy the condition $\nabla \cdot \tilde{\boldsymbol{\varepsilon}} \cdot \boldsymbol{E}=\boldsymbol{\nabla} \cdot \tilde{\boldsymbol{\mu}} \cdot \boldsymbol{H}=0$, the "degree of freedom" in essence is reduced to four arbitrary functions [17]. By proper choice of the auxiliary functions $\Psi_{e}$ in (9) and $\Psi_{m}$ in (12), one may represent the field in terms of two components of either $\pi_{e}$ and $\pi_{m}$ or in terms of one component of $\pi_{e}$ and another of $\pi_{m}$. The second possibility is considered in the next section.

It may be pointed out that the argument given by Bromwich [4] that a field in a homogeneous media may be represented, in general, in terms of two field components is not generally correct. His argument is that the field is identically zero if such components are zero. This reasoning obviously fails if one takes into account TEM wave type. The possibility of representing the field in more than one form in terms of Hertz potentials has many advantages. Some of these forms may yield a faster convergent field representation than the others [18] or may easily lead to some desired asymptotic expressions [22]. This aspect of the analysis, though very interesting, will not be considered in this work.

\section{Special Representations}

As pointed out in the previous section, a proper choice of the auxiliary functions $\Psi_{e}$ and $\Psi_{m}$ in (9) and (12), respectively, allows Hertzian vectors to be chosen which have four of their six components identically zero. In general, the differential equations satisfied by the two remaining components are of higher order than the second. The conditions required for the reduction of the order to the second is considered in this section. Let the field be represented in terms of the orthogonal curvilinear coordinates $q_{n}(n=1,2,3)$ defined by the metric

$d s^{2}=\sum_{n=1}^{3} h_{n}^{2} d q_{n}^{2}$

We assume that at every point of space the permeability and permittivity tensors have their principal axes in the direction of the coordinate axes with components $\mu_{n}$ and $\varepsilon_{n}$, respectively. Besides simplifying the analysis, this particular choice of $\tilde{\mu}$ and $\tilde{\boldsymbol{z}}$ is the same as that considered by Friedman [11] who dealt with the constant permeability case, thus allowing the comparison with his results. Such a choice also eliminates the confusion in the definition of TE(TM) mode since if $E_{n}=0\left(H_{n}=0\right)$ implies that $D_{n}=0$ $\left(B_{n}=0\right)$.

Consider the representation given by (7) to (9) with the Hertz vector having only one component, i.e.,

$\pi_{e}=\pi \hat{u}_{1}$

where $\hat{u}_{1}$ is a unit vector along the coordinate axis $q_{1}$. Substituting (16) into (9) leads to

$\varepsilon_{1} \ddot{\pi}-\frac{1}{h_{2} h_{3}}\left[\partial_{2}\left\{\frac{h_{3}}{\mu_{3} h_{1} h_{2}} \partial_{2}\left(h_{1} \pi\right)\right\}\right.$
$\left.+\partial_{3}\left\{\frac{h_{2}}{\mu_{2} h_{1} h_{3}} \partial_{3}\left(h_{1} \pi\right)\right\}\right]=\frac{\varepsilon_{1}}{h_{1}} \partial_{1} \Psi$
$\frac{1}{h_{1} h_{3}} \partial_{1}\left\{\frac{h_{3}}{\mu_{3} h_{1} h_{2}} \partial_{2}\left(h_{1} \pi\right)\right\}=\frac{\varepsilon_{2}}{h_{2}} \partial_{2} \Psi$

and

$\frac{1}{h_{1} h_{2}} \partial_{1}\left\{\frac{h_{2}}{\mu_{2} h_{1} h_{3}} \partial_{3}\left(h_{1} \pi\right)\right\}=\frac{\varepsilon_{3}}{h_{3}} \partial_{3} \Psi$

where $\partial_{n}$ represents differentiation with respect to the coordinate $q_{n}$. The equation obtained for $\pi$ on elimination of $\Psi$ is, in general, of higher order than the second. If, however, the condition

$h_{2} / h_{3}$ independent of $q_{1}$

holds, (18) and (19) reduce to

$\partial_{1}\left\{\frac{1}{\mu_{3} h_{1}} \partial_{2}\left(h_{1} \pi\right)\right\}=h_{1} \varepsilon_{2} \partial_{2} \Psi$

and

$\partial_{1}\left\{\frac{1}{\mu_{2} h_{1}} \partial_{3}\left(h_{1} \pi\right)\right\}=h_{1} \varepsilon_{3} \partial_{3} \Psi$.

Consideration of (21) and (22) leads to three conditions, in addition to (20), if any of which is valid, $\Psi$ may be determined in terms of a first derivative of $\pi$, thus reducing the order of the differential equation to be satisfied by $\pi$ to the second.

\section{Condition (a)}

If $\left(h_{1} \varepsilon_{2}, h_{1} \varepsilon_{3}, h_{1} \mu_{2}\right.$ and $\left.h_{1} \mu_{3}\right)$ are independent of $q_{2}$ and $q_{3},(21)$ and (22) become

$\partial_{12}\left(\frac{\pi}{\mu_{3}}\right)=\partial_{2}\left(h_{1} \varepsilon_{2} \Psi\right)$

$\partial_{13}\left(\frac{\pi}{\mu_{2}}\right)=\partial_{3}\left(h_{1} \varepsilon_{3} \Psi\right)$. 
Furthermore, if $\varepsilon_{2}=\varepsilon_{3}=\varepsilon$ and $\mu_{2}=\mu_{3}=\mu$, the function $\Psi$ satisfying (23) and (24) is then given by

$$
\Psi=\frac{1}{h_{1} \varepsilon} \partial_{1}\left(\frac{\pi}{\mu}\right)
$$

and the equation satisfied by $\pi$ is then given by

$$
\begin{aligned}
& \varepsilon_{1} \ddot{\pi}-\frac{1}{h_{2} h_{3}}\left[\partial_{2}\left\{\frac{h_{3}}{\mu h_{1} h_{2}} \partial_{2}\left(h_{1} \pi\right)\right\}\right. \\
& \left.+\partial_{3}\left\{\frac{h_{2}}{\mu h_{1} h_{3}} \partial_{3}\left(h_{1} \pi\right)\right\}\right]=\frac{\varepsilon_{1}}{h_{1}} \partial_{1}\left\{\frac{1}{h_{1} \varepsilon} \partial_{1}\left(\frac{\pi}{\mu}\right)\right\} .
\end{aligned}
$$

It may be noted that this case was previously considered by Nisbet [9] for isotropic media.

\section{Condition (b)}

If $\left(h_{1} \varepsilon_{2}\right.$ and $\left.h_{1} \varepsilon_{3}\right)$ are independent of $q_{1}$ and $\left(\mu_{2} \varepsilon_{3} h_{1}^{2}\right.$ $\left.=\mu_{3} \varepsilon_{2} h_{1}^{2}\right)$ is independent of $q_{2}$ and $q_{3}, \Psi$ is then given by

$\Psi=\partial_{1}\left\{\frac{\pi}{\mu_{3} \varepsilon_{2} h_{1}}\right\}$.

\section{Condition (c)}

The third situation leading to a second order differential equation for $\pi$ results if $\left(h_{1} \mu_{2}\right.$ and $\left.h_{1} \mu_{3}\right)$ are independent of $q_{1}$ and $\left(\mu_{2} \varepsilon_{3} h_{1}^{2}=\mu_{3} \varepsilon_{2} h_{1}^{2}\right)$ is independent of $q_{2}$ and $q_{3}$ in which case $\Psi$ is given by

$\Psi=\frac{1}{\mu_{2} \varepsilon_{3} h_{1}^{2}} \partial_{1}\left(h_{1} \pi\right)$

The analogous appearance of permeability and permittivity tensor components should be noted and it is apparent that this is a result of the duality between electric and magnetic quantities. As a consequence, the conditions to be satisfied for a TM-field representation are identical to those required for a TE-field representation.

It may be noted that Friedman [11] considered the present problem of mode decoupling when the permeability was assumed to be isotropic and constant. It appears that his theorem does not cover all the possibilities that were implied. In fact, Friedman's result corresponds only to case (a) above ${ }^{1}$.

Another class of problems for which $\pi$ satisfies a second order differential equation may be obtained

1 The author is indebted to Professor Lewin of the University of Colorado, Boulder, Colorado for a useful discussion on this point. when $h_{1}, h_{2}$ and $h_{3}, \tilde{\boldsymbol{\mu}}$ and $\tilde{\boldsymbol{\varepsilon}}$ as well as the solution are independent of one coordinate. This class of problems can be classified as:

A) Field is Independent of $q_{1}$

In this case $\pi$ satisfies the equation

$\varepsilon_{1} \ddot{\pi}-\frac{1}{h_{2} h_{3}}\left[\partial_{2}\left\{\frac{h_{3}}{\mu_{3} h_{1} h_{2}} \partial_{2}\left(h_{1} \pi\right)\right\}\right.$
$\left.+\partial_{3}\left\{\frac{h_{2}}{\mu_{2} h_{1} h_{3}} \partial_{3}\left(h_{1} \pi\right)\right\}\right]=0$.

This case was previously considered by Nisbet [9] with the medium assumed to be isotropic.

B) Field is Independent of $q_{2}$

In this case, if we let

$\frac{h_{2}}{\mu_{2} h_{1} h_{3}} \partial_{3}\left(h_{1} \pi\right)=\Pi$

we find that $\Pi$ satisfies the second order differential equation

$\mu_{2} \frac{h_{1} h_{3}}{h_{2}} \ddot{\Pi}-\partial_{3}\left\{\frac{h_{1}}{\varepsilon_{1} h_{2} h_{3}} \partial_{3} \Pi\right\}-\partial_{1}\left\{\frac{h_{3}}{\varepsilon_{3} h_{1} h_{2}} \partial_{1} \Pi\right\}=0$.

On the other hand, analysis of (18) and (19) leads to the following conditions under which $\pi$ satisfies a second order differential equation

B-1) If $\mu_{2} \frac{h_{1} h_{3}}{h_{2}}$ is independent of $q_{1}$ and $\mu_{2} \varepsilon_{3} h_{1}^{2}$ is a function of $q_{1}$ only, $\Psi$ is then given by

$\Psi=\frac{1}{\mu_{2} \varepsilon_{3} h_{1}^{2}} \partial_{1}\left(h_{1} \pi\right)$.

B-2) If $\mu_{2} \frac{h_{1} h_{3}}{h_{2}}$ and $\varepsilon_{3} \frac{h_{1} h_{2}}{h_{3}}$ are functions of $q_{1}$ only, $\Psi$ then has the form

$\Psi=\frac{h_{3}}{h_{1} h_{2} \varepsilon_{3}} \partial_{1}\left(\frac{h_{2} \pi}{h_{3} \mu_{2}}\right)$.

B-3) If $\varepsilon_{3} \frac{h_{1} h_{2}}{h_{3}}$ is a function of $q_{3}$ only while $\mu_{2} \varepsilon_{3} h_{1}^{2}$ is independent of $q_{3}$ in which case

$\Psi=\frac{h_{3}}{h_{1} h_{2} \varepsilon_{3}} \partial_{1}\left(\frac{h_{2} \pi}{h_{3} \mu_{2}}\right)$. 


\section{C) Field is Independent of $q_{3}$}

In this case, conditions similar to case B follows by interchanging the subscripts 2 and 3 .

Contrary to the first case discussed in this paper, and due to the two-dimensional character of the problem, conditions for TE-field expansion do not automatically yield those required for TM-field representation. The required conditions for the latter representation may be easily obtained by interchanging the components of $\tilde{\boldsymbol{\mu}}$ and $\tilde{\boldsymbol{\varepsilon}}$.

The discrepancies in the aforementioned theorem of Friedman should be obvious by now. In fact, condition (20) which is unnecessary for cases B and C above, is essential according to his analysis. According to his theorem under no condition can one represent the field in terms of a representation which is transverse to $\theta$ in spherical coordinates, which is incorrect if the field is independent on $\phi$ [18]. Using the same argument, Bromwich's analysis for homogeneous media [4], which is the basis of Friedman's theorem, does not cover all the possibilities.

It may be pointed out that different conditions may lead to the same $\Psi$, for example B-2 and B-3, but corresponding $\pi$ is, obviously, different. Depending on the coordinate system and the medium, whether it is isotropic or of constant $\tilde{\boldsymbol{\mu}}$ or $\tilde{\boldsymbol{\varepsilon}}$, the conditions derived above are not necessarily independent. Limiting our consideration to the cartezian, cylindrical and the spherical coordinate systems, conditions leading to representations transverse to one of the coordinates are given below.

\section{I) Cartesian Coordinates $(x, y, z)$}

Representation transverse to $x(T-x)$ is possible if any of the following conditions are satisfied

1) $\varepsilon_{y}=\varepsilon_{z}=\varepsilon(x)$ and $\mu_{y}=\mu_{z}=\mu(x)$

2) $\varepsilon_{y}=\varepsilon_{y}(y, z), \varepsilon_{z}=\varepsilon_{z}(y, z)$ and $^{2} \mu_{y} \varepsilon_{z}=\mu_{z} \varepsilon_{y}=f(x)$

3) $\mu_{y}=\mu_{y}(y, z), \mu_{z}=\mu_{z}(y, z)$ and $\mu_{y} \varepsilon_{z}=\mu_{z} \varepsilon_{y}=f(x)$

4) Field, $\tilde{\mu}$ and $\tilde{\boldsymbol{\varepsilon}}$ are independent of $x, y$ or $z$.

\section{II) Cylindrical Coordinates $(r, \phi, z)$}

a) Representation transverse to $r(T-r)$ is possible if the field, $\tilde{\boldsymbol{\mu}}$ and $\tilde{\boldsymbol{\varepsilon}}$ are independent of $\phi$ or $z$.

\footnotetext{
${ }^{2}$ In what follows, $f, g$ and $\tilde{f}$ represent arbitrary functions.
}

b) $(T-\phi)$ representation is admissible if any of the following conditions are satisfied:

1) $r \varepsilon_{r}=r \varepsilon_{z}=f(\phi)$ and $r \mu_{r}=r \mu_{z}=g(\phi)$

2) $r \varepsilon_{r}=f(r, z), r \varepsilon_{z}=g(r, z)$ and $r^{2} \mu_{r} \varepsilon_{z}=r^{2} \mu_{z} \varepsilon_{r}=\tilde{f}(\phi)$

3) $r \mu_{r}=f(r, z), r \mu_{z}=g(r, z)$ and $r^{2} \mu_{r} \varepsilon_{z}=r^{2} \mu_{z} \varepsilon_{r}=\tilde{f}(\phi)$

4) Field, $\tilde{\boldsymbol{\mu}}$ and $\tilde{\boldsymbol{\varepsilon}}$ are independent of $r, \phi$ or $z$.

c) $(T-z)$ representation is possible under any of the following conditions:

1) $\varepsilon_{r}=\varepsilon_{\phi}=\varepsilon(z)$ and $\mu_{r}=\mu_{\phi}=\mu(z)$

2) $\varepsilon_{r}=\varepsilon_{r}(r, \phi), \varepsilon_{z}=\varepsilon_{z}(r, \phi)$ and $\mu_{r} \varepsilon_{\phi}=\mu_{\phi} \varepsilon_{r}=f(z)$

3) $\mu_{r}=\mu_{r}(r, \phi), \mu_{z}=\mu_{z}(r, \phi)$ and $\mu_{r} \varepsilon_{\phi}=\mu_{\phi} \varepsilon_{r}=f(z)$

4) Field, $\tilde{\boldsymbol{\mu}}$ and $\tilde{\boldsymbol{\varepsilon}}$ are independent of $z$ or $\phi$.

III) Spherical Coordinates $(r, \theta, \phi)$

a) $(T-r)$ representation is possible if any of the following conditions are met:

1) $\varepsilon_{\theta}=\varepsilon_{\phi}=\varepsilon(r)$ and $\mu_{\theta}=\mu_{\phi}=\mu(r)$

2) $\varepsilon_{\theta}=\varepsilon_{\theta}(\theta, \phi), \varepsilon_{\phi}=\varepsilon_{\phi}(\theta, \phi)$ and $\mu_{\theta} \varepsilon_{\phi}=\mu_{\phi} \varepsilon_{\theta}=f(r)$

3) $\mu_{\theta}=\mu_{\theta}(\theta, \phi), \mu_{\phi}=\mu_{\phi}(\theta, \phi)$ and $\mu_{\theta} \varepsilon_{\phi}=\mu_{\phi} \varepsilon_{\theta}=f(r)$

4) Field, $\tilde{\boldsymbol{\mu}}$ and $\tilde{\boldsymbol{\varepsilon}}$ are independent of $\phi$.

b) $(T-\theta)$ representation is possible if the field, $\tilde{\mu}$ and $\tilde{\boldsymbol{\varepsilon}}$ are independent of $\phi$.

c) $(T-\phi)$ representation is admissible, if any of the following conditions are satisfied:

1) $\varepsilon_{r}=\varepsilon_{\theta}, \mu_{r}=\mu_{\theta}$ $r \sin \theta \varepsilon_{r}=f(\phi)$ and $r \sin \theta \mu_{r}=g(\phi)$

2) $h \varepsilon_{r}=f(r, \theta), h \varepsilon_{\theta}=g(r, \theta)$ and $h^{2} \mu_{r} \varepsilon_{\theta}=h^{2} \mu_{\theta} \varepsilon_{r}=f(\phi)$ where $h=r \sin \theta$

3) $h \mu_{r}=f(r, \theta), h \mu_{\theta}=g(r, \theta)$ and $h^{2} \mu_{r} \varepsilon_{\theta}=h^{2} \mu_{\theta} \varepsilon_{r}=f(\phi)$ where $h=r \sin \theta$

4) Field, $\tilde{\boldsymbol{\mu}}$ and $\tilde{\boldsymbol{\varepsilon}}$ are independent of $\phi$.

It may be pointed out that due to the singularity required for the medium parameters, the first three conditions of the $T-\phi$ representation for both cylindrical and spherical coordinates are not possible to realize.

\section{Conclusion}

This paper dealt with the general representation of electromagnetic fields in terms of electric and magnetic Hertz vectors. Conditions for uncoupling 
the TE- and TM-modes were given for media whose permeability and permittivity tensors have only diagonal elements. Admittedly, some of these conditions may lead to impractical situations, as far as realizability is concerned, in which case one has to use a more complicated analysis. Because of our assumption with respect to $\tilde{\mu}$ and $\tilde{\varepsilon}$, the results do not include the most general type of anisotropy and, in particular, those of plasma and ferrite media. As pointed out before, the condition for mode decoupling under a mixed boundary condition as well as decoupling via coordinate transformation, even for the isotropic case, deserves further investigation. When the problem admits more than one representation, the relative convergence of one representation compared to the other and the relative usefulness of such representation as far as the derivation of asymptotic behaviors of the field were studied only in some particular cases and is not yet fully investigated.

The conditions for separability of coordinates for the equation satisfied by Hertz potentials in homogeneous isotropic media are well documented [23]. Extension of such study to the inhomogeneous case is under investigation.

Acknowledgements. The author wishes to acknowledge the valuable and stimulating discussions with Professor C-M. Chu and the comments of Professor R. E. Hiatt and Professor C-T. Tai.

This research was supported by NASA under Grant NGR-23005-477.

\section{References}

1. H. Hertz: Ann. Physik 30, 1 (1889)

2. E.T. Whittaker: Proc. Lond. Math. Soc. 1, 367 (1903)

3. P.Debye: Ann. Physik (4) 30, 57 (1909)

4. T.J.I'A.Bromwich: Phil. Mag. 38, 143 (1919)

5. W.W. Hansen: Phys. Rev. 47, 139 (1935)

6. H.Bremmer: Terrestrial Radio Waves (Elsevier, New York, etc. 1949), pp. $138-141$

7. C-T.Tai: Appl. Sci. Res. B7, 113 (1958)

8. A. Nisbet: Proc. Roy. Soc. A 231, 250 (1955)

9. A. Nisbet: Proc. Roy. Soc. A 240, 375 (1957)

10. W. H. McCrea: Proc, Roy. Soc. A40, 447 (1957)

11. B.Friedman: "Propagation in a non-homogeneous medium"; Electromagnetic Waves, R. Larger (editor) (The University of Wisconsin Press, Madison 1962), pp. 301-309

12. A.Wexler: IEEE Trans. Microwave Theory Techniques MTT-13, 875 (1965)

13. P.L.E. Uslenghi: Elect. Letters 3, 400 (1967)

14. R.G. Parkinson: Elect. Letters 5, 182 (1969)

15. P.E. Bisbing: IEEE Trans. Antennas Prop. AP-20, 217 (1972)

16. L. S. Benenson, Yu.A.Zaytsev: Radio Eng. Elect. Phys. 16, 405 (1971)

17. R.K. Luneberg: New York University, Math. Res. Group. Res. Rpt. No. 172-6, Jan. 1948

18. N.Marcuvitz: "Field representations in spherically stratified regions"; The Theory of Electromagnetic Waves, M. Kline (editor) (Interscience, New York 1951) pp. 263-316

19. H. S.Green, E. Wolf: Proc. Phys. Soc. A66, 1129 (1953)

20. S. N. Samaddar: J. Franklin Inst. 276, 225 (1963)

21. L.B. Felsen, N. Marcuvitz: Radiation and Scattering of Waves (Prentice Hall, Englewood Cliffs N. J. 1973) pp. $24-25$ and $195-202$

22. A. Erteza, B. K. Park: IEEE Trans. Antennas Prop. AP-17, 376 (1969)

23. P.M.Morse, H.Feshbach: Methods of Theoretical Physics (McGraw-Hill, New York, etc. 1953) pp. 495-522 